\title{
Projet-pilote «Trajectoire intersectorielle des patients atteints du cancer du côlon»
}

\author{
Varja Meyer ${ }^{a}$, \\ Jürg Nadig, ${ }^{b}$ \\ Jürg Pfisterer', \\ Christoph Bosshard ${ }^{d}$
}

a lic.phil.I, cheffe suppléante de la division DDQ, Responsable opérationnelle de l'ASQM

b Dr, président SSOM

c Dr, Co-Président SSMI

d Dr, membre du Comité central de la FMH, Responsable du département DDQ et de l'ASQM
Correspondance:

Varja Meyer

FMH / Académie suisse pour

la qualité en médecine (ASQM)

Elfenstrasse 18

CH-3000 Berne 15

Tél. 0313591111

Fax 0313591112

asqm[at]fmh.ch

\section{Contexte}

Les progrès de la médecine et la spécialisation professionnelle augmentent le nombre d'intervenants spécialisés impliqués dans les diverses étapes d'un traitement. Les patients sont ainsi soit ponctuellement, soit parallèlement, en rapport avec différents spécialistes au cours de leur prise en charge. En règle générale, chacun de ces traitements s'appuie sur les connaissances scientifiques actuelles, telles que décrites dans les guidelines. Pour garantir un accompagnement compétent des patients et afin d'éviter les doublons ainsi que les retards inutiles au traitement, la coordination des différentes activités revêt toujours plus d'importance, en particulier pour les procédures thérapeutiques complexes.

Le présent projet-pilote de l'ASQM met explicitement l'accent sur le développement d'une trajectoire intersectorielle du patient prenant aussi bien en compte les deux secteurs (ambulatoire/stationnaire) que les différentes institutions et disciplines concernées. Comme le développement de trajectoires intersectorielles en est encore à ses débuts, il existe peu de données probantes aptes à augmenter leur efficacité et à améliorer la qualité des soins. Dans le cadre de ce projet-pilote, il s'agira donc d'acquérir des expériences et de réunir des informations pour les fournisseurs de prestations et les patients concernant les bénéfices et les inconvénients d'une structure thérapeutique interprofessionnelle et intersectorielle dans le cas d'un tableau clinique aussi complexe et multidisciplinaire avec de nombreuses interfaces.

\section{Focus: cancer du côlon}

D’une manière générale, les spécialistes et les patients en oncologie sont tributaires d'une collaboration interdisciplinaire la mieux à même de répondre à leurs besoins. En effet, le traitement de ce cancer comme de beaucoup d'autres demande l'intervention de spécialistes issus de domaines médicaux et de groupes professionnels très divers. C'est aussi la raison pour laquelle le «Programme national contre le cancer pour la Suisse 2011-2015» et le projet de rapport «Stratégie nationale contre le cancer» accordent beaucoup d'importance à la trajectoire du patient. Le présent projet-pilote se concentrera exclusivement sur le cancer du côlon. Les expériences acquises au cours de ce projet serviront de base au développement d'autres trajectoires intersectorielles du patient.

\section{Objectifs du projet}

Les principaux objectifs du projet-pilote sont les suivants:

- Mettre à disposition des outils permettant de garantir qu'un patient atteint du cancer du côlon reçoive un traitement de qualité élevée, standardisé et coordonné de manière optimale, fondé sur des guidelines (inter)nationales reconnues, indépendamment de son lieu de domicile.

- Acquérir des expériences précieuses concernant les bénéfices et les inconvénients d'une part de l'élaboration de trajectoires intersectorielles avec de nombreuses interfaces et d'autre part de la collaboration interdisciplinaire qui en découle.

S'il s'avère possible de développer et d'implémenter avec succès une trajectoire-pilote dans différents secteurs et disciplines, d'autres travaux pourront être entrepris lors d'une prochaine étape, par exemple l'adaptation de la trajectoire à d'autres régions de soins, l'élaboration de trajectoires pour d'autres tableaux cliniques ainsi que l'actualisation de la trajectoire dans un intervalle à définir.

\section{Intérêts du projet}

Le projet-pilote vise notamment à vérifier les hypothèses suivantes:

- La probabilité qu'un patient bénéficie d'un traitement de qualité élevée, fondé sur des guidelines reconnues et des standards de traitement définis - indépendamment de l'accès au système - augmente.

- La prise en charge intégrée du patient au-delà des interfaces est encouragée.

- La collaboration interdisciplinaire au sein du corps médical et avec d'autres groupes professionnels impliqués est renforcée.

- En définissant une trajectoire intersectorielle de portée générale, les sociétés de discipline médicale et les groupes professionnels posent les jalons devant permettre de fournir un traitement de qualité élevée aux patients atteints du cancer du côlon.

- Grâce à la définition de standards de traitement, les différents fournisseurs de prestations peuvent plus facilement motiver les mesures thérapeutiques envers les assureurs.

- La trajectoire permet de diminuer les coûts de traitement en évitant les doublons et les retards. 
- Le projet-pilote crée des conditions aptes à simplifier l'identification de la qualité des soins pour une maladie en particulier.

- Les travaux de base concernant la méthodologie et la mise en œuvre ainsi que les expériences réalisées peuvent être mis à profit en vue de développer et de mettre en ouvre des trajectoires portant sur d'autres maladies.

\section{Large soutien}

Pas moins de 20 sociétés de discipline médicale et groupes professionnels impliqués dans le traitement de patients atteints du cancer du côlon ont rejoint le projet. Il s'agit d'une part des sociétés des disciplines concernées, et d'autre part des différentes professions non médicales impliquées comme les soins oncologiques, la stomathérapie et la diététique. La délégation officielle des membres de l'équipe de projet par les organisations professionnelles concernées permet de garantir l'assise du projet sur le plan scientifique et politique. Pour planifier et concrétiser le projet au mieux du point de vue de la méthode et pour intégrer le savoir-faire international, l'ASQM collabore étroitement avec l'European Pathway Association.

\section{Intégration des éléments existants}

Pour éviter les doublons et utiliser au mieux les synergies, la direction du projet a procédé au début
2014, soit avant le lancement officiel du projet, à une enquête auprès de l'ensemble des groupes professionnels et responsables d'établissements de formation postgraduée des hôpitaux suisses concernés pour savoir s'il existe déjà des trajectoires, des guidelines, des normes de traitement et du matériel d'information pour les patients atteints du cancer du côlon. Cette enquête a permis de réunir de précieuses informations qui seront dans la mesure du possible intégrées dans les travaux.

\section{Direction du projet}

La direction du projet se compose des personnes suivantes:

- Varja A. Meyer, lic. phil., FMH / Académie suisse pour la qualité en médecine (ASQM)

- Dr Jürg Nadig, Société suisse d'oncologie médicale (SSOM)

- Dr Jürg Pfisterer, Société suisse de médecine interne générale (SSMI)

\section{Contact}

Souhaitez-vous en savoir plus sur le projet-pilote «Trajectoire intersectorielle des patients atteints du cancer du côlon»? N'hésitez pas à prendre contact avec nous à l'adresse asqm[at]fmh.ch ou au numéro 0313591111

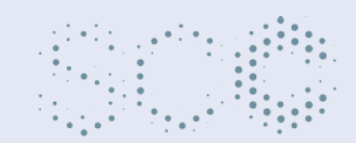 \\ SWISs cancer congress \\ Premier Congrès suisse du cancer Le 4 septembre 2014, Centre Paul Klee, Berne}

En Suisse, une personne sur trois est atteinte d'un cancer au cours de son existence. Les patients sont généralement pris en charge par des professionnels de différentes disciplines: médecins, soignants, radio-oncologues, chirurgiens, psycho-oncologues, professionnels de la réhabilitation ou de la palliation, etc. La collaboration de tous ces professionnels est depuis toujours étroite et s'intensifiera encore à l'avenir. C'est la raison pour laquelle nous sommes persuadés qu'un congrès commun réunissant tous ces prestataires serait souhaitable.

Le 4 septembre 2014 se déroulera le premier Congrès suisse du cancer au centre Paul Klee de Berne. Tous les médecins, soignants et professionnels intervenant dans la prise en charge des patients cancéreux sont invités à participer, quel que soit leur point d'intervention, du diagnostic à la palliation. Nous espérons aussi que l'ensemble du territoire suisse sera représenté, et notamment que la génération montante de médecins et de soignants sera présente en nombre. Le congrès portera sur les thématiques suivantes:

Parcours thérapeutique, illustré par l'exemple du projet pilote de l'Académie suisse pour la qualité en médecine «Trajectoire intersectorielle des patients atteints du cancer du côlon» : les trajectoires intersectorielles améliorent-elles les résultats cliniques ou servent-elles d'abord à optimiser les coûts? Quelles opportunités et quels risques leur sont-ils liés?

- Collaboration interdisciplinaire / interprofessionnelle: comment relier des maillons toujours plus nombreux? Comment les tumor-boards améliorent-ils la collaboration? Comment relever au mieux les défis de l'interdisciplinarité? Quelles sont les solutions neuves à l'horizon?
- Certification et registre: quelle est la plus-value des certificats et registres de qualité? Quelles opportunités et quels risques leur sont-ils liés?

- Une profession en mutation: l'évolution de la société et du cadre législatif (LPMéd, etc.) modifie la profession de tous les intervenants en oncologie, qu'ils soient médecins, soignants ou autres professionnels: à quoi faut-il nous attendre?

- Recherches sur l'offre de soins: Où en sommes-nous aujourd'hui et où va-t-on? Les trajectoires intersectorielles permettront-elles d'améliorer la qualité des soins? Quels sont les projets en cours en Suisse?

Les sujets seront présentés par des scientifiques et des spécialistes de politique de santé suisses et étrangers. Par ailleurs, les porteurs de la Stratégie nationale contre le cancer feront le point sur cette stratégie et présenteront les projets actuels.

Le Congrès suisse du cancer est une nouvelle plateforme dont le but est de promouvoir la mise en réseau et l'échange d'expérience des médecins, soignants et autres professionnels intervenant dans le traitement et la prise en charge des patients atteints de cancer.

Plus d'informations sur le congrès (programme, inscription en ligne) sous www.swisscancercongress.ch.

Le comité scientifique: PD Dr Judith Alder (SSPO), judith.alder[at]psychologie. ch; Mme Irène Bachmann-Mettler (SOS), irene.bachmann[at]gmx.net; Prof. Markus Borner (SSOM), markus.borner[at]me.com; Dr Stephan Eberhard (oncoreha.ch), stephan.eberhard[at]gmail.com; PD Dr Günther Gruber (SRO), guenther.gruber[at]hirslanden.ch; Dr Jürg Nadig (SFSM), juerg.nadig[at] hin.ch; PD Dr Florian Strasser (palliative.ch), Florian.Strasser[at]kssg.ch; Dr Markus Trutmann (fmCh), markus.trutmann[at]fmch.ch 